\title{
Future Trends of the Healthcare Data Predictive Analytics using Soft Computing Techniques in Data Science
}

\author{
Dr. Sasikala Dhamodaran ${ }^{1}$, Archana Balmoor ${ }^{2}$ \\ ${ }^{1}$ Professor, CVR College of Engineering/CSE Department, Hyderabad, India \\ Email: anjansasikala@cvr.ac.in \\ ${ }^{2}$ PG Scholar, CVR College of Engineering/ CSE Department, Hyderabad, India \\ Email: archanabalmoor@gmail.com
}

\begin{abstract}
Predictive Analytics, Soft Computing (SC) and Optimization, Data Mining and Data Science are rapidly becoming some of the most-discussed, perhaps utmost glorified topics in healthcare business. Artificial Intelligence, Machine Learning, Artificial Neural Networks, Fuzzy Logic, Expert Systems, etc., is well-studied disciplines with a long history of success in many industries. Healthcare can acquire treasured sessions from this prior achievement to startup the efficacy of predictive analytics for refining patient care, chronic disease management, hospital administration and supply chain efficiencies. The prospects that presently occurs for healthcare systems is to state what "predictive analytics" stands for to them and how can it be cast off furthermost excellently to cause further enhancements.

In all industries including healthcare, prediction plays a best worthwhile role when that data is passed on as accomplishments. The inclinations to mediate the vital data is in harnessing the power of historical and real-time data with visions from forecasting those data based on the times ahead. Importantly, to best gauge efficacy and value, both the predictor and the intervention must be integrated within the same system and workflow where the trend occurs.

A valuable report of the organized publicity and expectation of predictive analytics in healthcare through a blend of psychology, digital technology, and entrepreneurship is available for real-time implementation for the good of the public. Review and evaluation on these disciplines pave ways to open up new arenas envisaging the future trends of Predictive analytics, Data Mining and Science and Soft Computing (SC) in healthcare, stepping strongly into pervasive computing, ambient intelligence, ubiquitous computing and many more automated technical concepts and computing's ahead.
\end{abstract}

Index Terms: Predictive Analytics, Soft Computing, Optimization, Healthcare, Artificial Intelligence, Machine Learning, Artificial Neural Networks, Fuzzy Logic, Expert Systems, Data Mining, Data Science

\section{INTRODUCTION}

Data is a collection of standards of studies with regards to qualitative or quantitative variables. Data and information or knowledge are frequently swapping within each other; yet data becomes information once it is detected in context or in support exploration.

Data science is a concept to unify abundant hypothetical authorities or proficient fields in a button hole to an issue or challenge that aids, technical procedures, methods, algorithms and approaches to unearth information and intuitions from information in several manners, together as structured extrapolation that comprehends a mixture of statistical practices from data mining, predictive modelling, and machine learning, that evaluate recent and past realities to formulate prophecies going on for the time ahead or else strange trials and vague, related to data mining online.

Predictive analytics is the exercise of mining data beginning from prevailing information suites in the direction to govern frameworks and visualize the forthcoming endings and inclinations [1].

The passionate post is just about the three explanations Why Comparative Analytics, Predictive Analytics and Natural Language Processing (NLP) hope for resolving Healthcare's Complications. Then retell that general catchphrases and hot topics constantly originate and go off. Identical to the up-to-date Hollywood trends that are growing and dropping, technological themes such as big data, bioinformatics, predictive analytics or genomic medicine are blended in and out create impact on retailing the summons, capital schemes, publication items and blogs for a limited time and then societies transfer on to the subsequent massive feature. Abundant of this "be on fire and reflects" can get up from the apparent be fond of concern by means of innovative technology. Globally, presently there are readily available bulky naïve data and additional materials are engendered timely on its own than may perhaps be captivated in a whole lifespan [2].

Soft Computing (SC) is an evolving arena that involves the harmonizing foundations of perceptrons, artificial intelligence, rough sets, fuzzy logic, SVM, Genetic algorithms, Bayesian network, neural computing, evolutionary computation, machine learning, expert systems, robotics, nature inspired computings and probabilistic reasoning. Owing towards their robust, deep, transfer, reinforcement, deep adversarial learnings, Q-learning, temporal difference learning and hybrid learning algorithms and reasoning capabilities, tolerance and upright acceptance of vagueness, fuzziness, ambiguity, fractional truth, guesstimate and inexactitude, soft computing practices, devise an extensive purposes across the globe turning them into applications with ambient intelligence in doing so crafting pervasive, multimedia, distributed, spatial and geographic, time series and sequence and ubiquitous functions. Thus, fixing the groundwork for a time ahead everywhere keeps people and machines stay fastened 
together. They will help in the improvements of human quality of life [3].

\section{Data Predictive Analytics}

Data Predictive Analytics states about the structured and unstructured data. Structured data is ordered fascinated by a configured storehouse, usually a databank, subsequently that the aforementioned constituents can turn into a storage component in which all positions can be distinctly gain access to by means of a specific database intended for further robust, efficient and effective evaluation, handling of input, process, output, and assessment. A data structure is a form of storehouse that puts in order data and evidence for that resolution.

Unstructured data (information) is evidence whichever doesn't encompass an established, well advanced with data model otherwise remains unorganized in a ready-made style. Unstructured information is stereotypically text-heavy, then possibly choose to hold data such as dates, numbers, and facts in addition.

From Figure 1. Predictive Analytics involves Structured and / or Unstructured data from a configured storehouse, usually a databank performing predictions, mining, analysis, evaluation, modeling, testing, deployment and reporting.

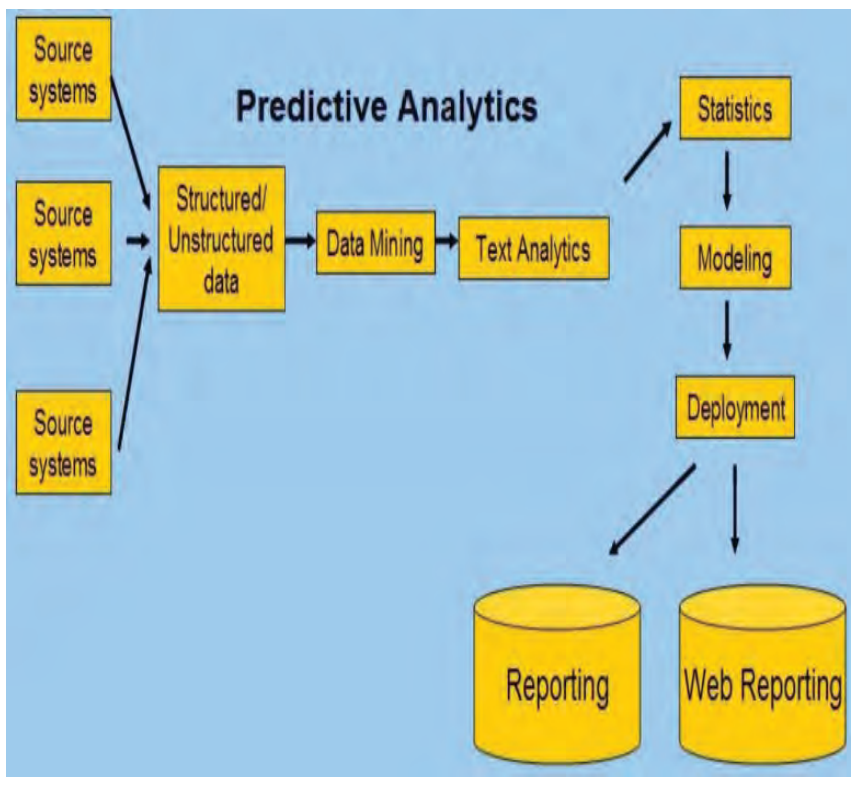

Figure 1. Predictive Analytics Detailed Process Flow

As of Figure 2. Predictive Analytics value chain indicates that based on the time and functions data can be analyzed, monitored and/or predicted established on actions that embraces what happened, why that happened, what is happening then and what is going to happen in future.

Predictive Analytics value chain aids many algorithms from Data Science, Data Mining, Statistics, Soft Computing, etc., bringing together the Management, Information Technology, Modeling Business Process, Decision Support Systems, etc., rendering Predictive Intelligence identifies trendy risks and opportunities time ahead in business and technology effectively interpreting big data for their benefit by assigning score remain subjective in this regard [4].

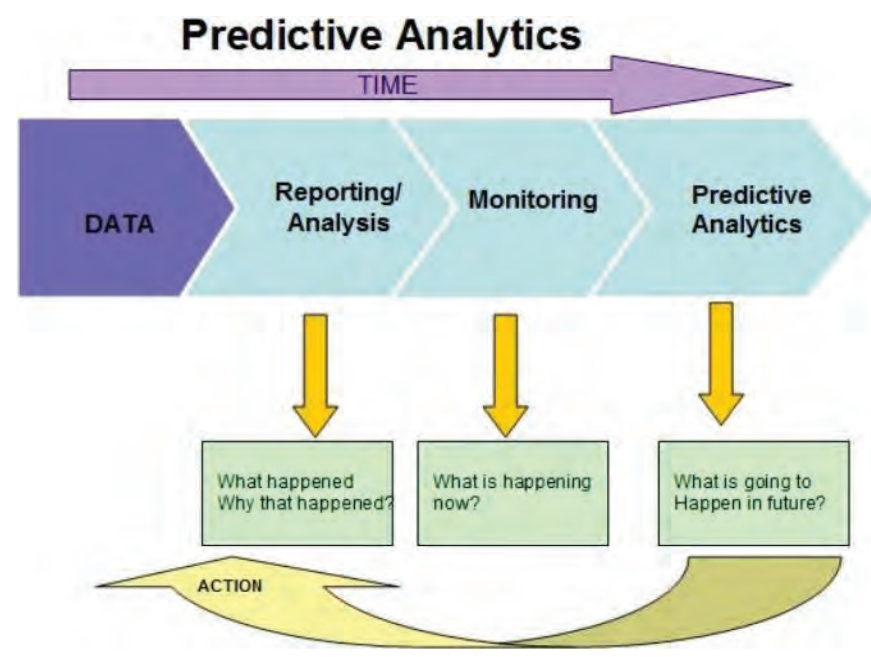

Figure 2. Predictive Analytics Value Chain

\section{A. Advantages of Data Predictive Analytics}

Basically, with its numerous forms as predictive mining and modeling, decision analysis and support, optimization, business reporting, predictive search, etc., widely, predictive analytics is pragmatic to a series of commercial approaches and a crucial team member in exploration of marketing promotions, and endorsement mechanisms delivering administrators and directors with policymaking means to impact upselling, auctions, sales and income anticipations, trade optimization, constant innovative merchandise progress, etc.

\section{B. Disadvantages of Data Predictive Analytics}

With all these values still, predictive analytics remain uncommon for one and all. Also, occasionally huge data collections remain tough to derive solutions. Few specify that predicting individual actions machines and methods decline due to study variables that are varying on or after fluctuating conditions to attitudes to associations to tempers that may change human behavior archetypes. Time to enact a share out just how fit these methods succeed. However, an archetype effective on same period, individual deeds deviates through time and hence the simulations too in essence to be streamlined just in time. The risk of economics is amalgamated with them here. Hence, only, data scientists, bolster an in-depth intelligence of predictive analytics be able to assist us in business forecasting, influencing when to and when not to realize predictive approaches into a scientific, administrative and technological know-how, schedule, handle and thriving best.

\section{Healthcare Data Predictive Analytics}

Predictive analytics and soft computing in healthcare transpire swiftly flattering as it is the supremely pondered topic of current value, possibly extremely-glorified issues in healthcare analytics. Soft Computing is a fortunately-researched branch of learning by means of an extensive past chronicle of accomplishments in numerous trades. Healthcare can acquire appreciated experiences on or after earlier victory to startup the efficiency of predictive analytics for shooting up patient care, unceasing ailment administration, clinic management, and supply chain 
proficiencies. The concern now for healthcare systems is to describe what "predictive analytics" signifies and in what manner it might be cast off utmost efficiently to cause enhancements. In healthcare, prediction is the utmost beneficial factor once that intelligence is turned over into accomplishments.

Mostly a great deal of Healthcare Data Predictive Analytics is aggrandizing the actuality of the state of affairs by means of deceptive or extraneous data that possibly will ascend on or after our apparent worship status quo through this innovative expertise. That is, the expertise is nearby, then again, the technology suffers from resources to distribute and infer real-time data hitherto on the aspect to be entirely technologically advanced.

Predictive Analytics catchphrase illness probably resolves to endure to grow and drop. Regrettably, deficient of appropriate substructure, recruitment and supplies to perform the minute a bit is prophesied with extreme conviction to materialize but drop rapidly due to the complete capability of connecting significant past, present and future inclinations and blueprints or styles in patient data. Expressed in different words that, deprived of the determination and drive for medical intrusion, any analyst - no significance how trustworthy it is will not be wholly operated.

The health catalytic agent is not alone with suitable technological context and metadata in the dwelling, then must similarly advance with new prescriptive analytics. Additionally, bond with each one and only facts prudently to medical primacies and quantifiable measures that includes price efficacy, medical etiquettes or patient consequences.

For flourishing, medical affairs forecasting and ensuing intrusion had better be mutually content and clinician piloted. Further precisely, embrace proof, endorsements and activities for each foreseen class or upshot.

Healthcare corporate, medical, and technological substantiations had better think of the identical final aspiration to make the most of the efficacy of the past, present and future inclinations for advancing patient care.

Confined via Health Catalytic agent, data modeling and algorithm evolutions are being explored using Healthcare Predictive Analytics using soft computing techniques in Python Programming language and its related automated tools. In progress tasks include classification models for a generic interpreter of clinic reentries, heart disaster, span of admission and clustering of patient conclusions to historical associates during allow-in-hospital. Furthermost significantly, core interlink to masses of de-acknowledged sickbay archives together in the inpatient and outpatient sites and elders and children populaces. This training data is vital to deal with the predictive analytics requests of customers and location customization. Consequently, as soon as the appeal arrives, whether it includes classification or clustering or feature selection, Health Catalytic agent brings forth, the tools and the data and the technology to well distribute highest executing predictive analytics.

\section{A. Advantages of Healthcare Data Predictive Analytics}

The economy of predictions. Shooting up patient care despite the fact preventing economic and repayment forfeits for sickbays.

\section{B. Disadvantages of Healthcare Data Predictive Analytics}

The societal dispute is leftovers: academic - industry productive ideas orbits to abundantly technologically advanced and employed executions in a real-time clinical aforesaid situation. Besides predictive analytics be cast off in serving cost controls and enrich patient care.

"What You See is What You Get" is both an advantage as well as a disadvantage. It is demanded as an advantage due to the reasons discussed below: Corroborate-centered drug is an authoritative means to support lessening therapy alternatives and unanticipated prices. Elite-system strategies impact more to the aspirations of systematized patient upshots and regulating expenses.

"To think in terms of a year, conceal a seed; in terms of 10 years, conceal trees; and in terms of 100 years, educate the individuals as much as possible." For the endurance victory of predictive analytics in healthcare, it's essential to carry out all of the above-mentioned practices. Still, the method can be on track on or after realizing from more corporate and technological know-how.

Auspiciously for healthcare, there remain several prevailing representations commencing from more businesses that are extremely resourceful at-risk stratification in the province of inhabitants managing. Correspondingly, the arithmetical effort of actuaries in the duty of handling populace life insurance risk and payout is as well meticulously recognized. Ahead of business knowledge, learning past will be anticipated to comfort certain possible troubles and drawbacks that can go with healthcare's approval of predictive analytics. Specified that predictive analytics are registered and obtainable of the probable stages scheduled on the Healthcare Analytics Adoption Model, about plentiful solutions and drawbacks that can arise at such a stage if not accurately arranged. Main instructions are itemized beneath:

1. Further data doesn't connect to extra acumen.

2. Awareness and worth are different.

3. Capability to infer information differs founded on the facts itself.

4. The execution itself can ascertain a trial.

For the healthcare industry, similar to further businesses, predictors resolution continuously stays extra benefits in the context of an added wide-ranging series of data, where the information can be wholly influenced to accomplishments.

The four vital instructions learnt for approving predictive analytics in healthcare are given as follows:

1. Precise Outperforms Total: Don't complicate further information through added awareness.

2. Unified Forecast: Don't complicate recognized by means of worthiness.

3. Stage of Conviction: Don't overvalue the capability to infer the information.

4. Status of the Business; Don't underrate the trial of execution.

Health Catalytic agent's new Soft Computing result prepares SC in healthcare standards, operational, and pervasive through three openings:

catalytic agent.sc - our SC representations and policy for construction of SC with wholly Health Catalytic agent. 
healthcare.sc - our way of inspiring the approval of SC in healthcare done free, open-source SC software that centralizes SC by letting down blocks passing.

Healthcare analytics platform - the first pillar (basis) for SC.

Health Catalytic agent Predictive Analytics and SC Artefacts and Facilities that are to be implemented includes the following:

- catalyst.sc: Health Catalytic agent SC Result

- healthcare.sc: Health Catalytic agent Open Source SC Toolset

- Health Catalytic agent Late-Binding Data Warehouse

- Health Catalytic agent Predictive Analytics Uses

- Vital Link Connected to Arteries Infection Avoidance Progressive Use

- Employment Administration Surveyor

- Chronic Disruptive Pulmonic Disease

- Patient Stream Surveyor

- Health Catalytic agent Facilities

\section{SOFT COMPUTING TECHNIQUES}

Though Soft computing was introduced earlier, it's currently practiced vigorously for solving complex real-time problems. It dispenses with rough set patterns, contrasting hard computing, is accepting fuzziness, vagueness, incomplete truth, and guesstimates. In operation, the pattern for soft computing is the human mind and is built on practices such as artificial neural networks, perceptron's, artificial intelligence, rough sets, fuzzy logic, SVM, Genetic algorithms, Bayesian network, machine learning, expert systems, robotics, nature inspired computing's and probabilistic reasoning.

Artificial neural networks: Artificial neural networks or connectionist systems are computing systems stimulated by the organic neural networks that establish instinctive brains. The neural network itself is not an algorithm, but reasonably a framework for numerous dissimilar mechanisms learning processes to operate as one and handle multifaceted data inputs.

Perceptron's: Computer archetypes or computerized machine created to signify or put on the capability of the brain to identify and differentiate.

Artificial intelligence: Intellect established by technologies, in distinction to the normal brainpower exhibited by individuals and more creatures.

Rough sets: An official rough calculation of a hard set in relationships of a pair of collections that deliver the lower and the upper estimate of the primary set.

Fuzzy logic: A method of multi-valued logic in which the truth values of variables could be any actual number amid 0 and 1 including them take up to hold the idea of incomplete truth, where the truth value may vary amongst wholly true and wholly false.

Support Vector Machine (SVM): Supervised learning models with related learning processes that investigate data applied for classification and regression analysis.

Genetic algorithms: Metaheuristic motivated by the procedure of natural selection that fits into the bigger class of evolutionary systems.
Bayesian network: Probabilistic graphical archetype that signifies a series of variables and their conditional enslavements through a directed acyclic graph (DAG).

Machine learning (ML): Technical learning systems and arithmetical patterns that computer systems practice to efficiently accomplish a precise work deprived of open commands, trusting on designs and interpretation as a substitute realized as a subdivision of artificial intelligence.

Expert systems: A part of software that utilizes catalogues of professional information to present intelligence or extend verdicts in such subjects as medical diagnosis.

Robotics: The division of knowledge that dispenses with the design, creation, operation, and use of robots.

Nature inspired computing's: Actual innovative subject that attempts to build up novel calculating methods in perceiving in what manner naturally happening incidents act to resolve complicated issues in numerous ecological circumstances. and

Probabilistic reasoning: The purpose of a probabilistic logic is to combine the capacity of probability theory to handle uncertainty with the capacity of deductive logic to exploit the structure of formal argument. The result is a richer and more expressive formalism with a broad range of possible application areas.

\section{A. Advantages of using Soft Computing Techniques}

$\mathrm{SC}$ is essential to attain multifaceted resolutions and select the excellent result as of numerous opportunities, via complicated systems.

SC involves speedy handing out influence and bulky memory that are just obtainable at a very small price.

Internet of Things (IoT) hypothesis initiated the need in imminent native, engineering and profitable markets for SC, demanding super-fast microcontrollers. Utilization of fuzzy logic, artificial neural networks, and expert systems in numerous normal household applications, such as washing machines, cookers, and fridges. Several industrial and commercial applications of soft computing are likewise in daily use predictable to arise in the following period. It is learnt that the soft computing theory and techniques and its applications is growing rapidly together with the use of IoT devices in future domestic, industrial and commercial markets.

\section{B. Disadvantages of using Soft Computing Techniques}

Incorrect learning algorithms and erroneous neural network architectures cause hindrances for the users. Numerous business experts are suffering from a lot of difficulties with neural network training. Besides this, bulky network is not capable to act on appropriateness to the innovative prototypes which were not operated for training.

\section{Data Science in HealthCare}

Data science is a diverse domain with multi-subjects intermingling and data interpretation, system promotion, and knowledge in solving logically multifaceted issues. At the central is the data. Storage of raw evidence, flowing in and warehoused in the enterprise data warehouses. Plentiful to study through mining it. 
Captivating a complete interpretation, plainness, appealing medical decision and constructing associations castoff in employing data science in healthcare [5].

Medicine detection, wearables, diagnostics, community health, lessened healthcare charges, best employment remain some of the foremost use cases wherever data science is causing a vast transformation in the healthcare industry followed by medical image analysis, genetics and genomics, manufacturing drugs, simulated support for patients and customers, predictive drug: forecast and analytical support, managing customer data and industry knowledge follow these.

- Developments in expertise

- Evolution of digital consumerism

- The necessity to contest growing prices - enhancing healthcare modernization.

Data mining embraces countless possibilities for the healthcare industry allowing health systems to methodically practice data and analytics to recognize inadequacies and excellent procedures that enhance healthcare and lessen the charges. Some specialists trust the chances to progress care and decrease expenses simultaneously may well employ to as considerable as $30 \%$ of total healthcare expenditure. This can be a win/win general. Then, owing to its difficulty and a sluggish degree of expertise, implementation, healthcare business, holdups after the others in instigating active data mining and analytic approaches.

Similar to analytics and business intelligence, the data mining too connote unusual facts to dissimilar individuals. Data mining is the study of big data sets to realize designs and practice those to estimate or forecast the probability of forthcoming proceedings.

Implementing the three systems - the analytics system, the best practice system and the adoption system is the crucial power in practical enhancement with any analytics lead in healthcare.

\section{A. Advantages of Data Science in Healthcare}

1. Seller's knowhow and special focus on healthcare.

2. SC pattern's get into widespread data bases.

3. SC pattern's affluence of operation.

4. SC pattern's understandability and get-in.

5. SC pattern's acknowledged performances with confidentiality guidelines.

\section{B. Disadvantages of Data Science in Healthcare}

1. Relative data doesn't push development.

2. Predictive Analytics fall short to contain results.

3. Variances in Healthcare Industry data confine the efficiency of Natural Language Processing (NLP).

4. A Structured method: advancing up the Diagnostic acceptance standard.

\section{Existing Healthcare Data Predictive ANALYTICS USING SOFT COMPUTING TECHNIQUES IN DATA SCIENCE}

Data Mining and Data Science perform a vital part in healthcare. Though therapeutic data are inestimable and very wealthy in information, but numerous times the valuable information perhaps go waste as it is failing to obtain the fruitful data from it. Abstraction of real data from rich healthcare data and rendering treasured the results for forecasting the illnesses slowly alter into rudiments. Data mining practices are employed to mine the enormous therapeutic data and to study the unseen design and associations that aids in achieving active evaluations.

A primary blueprint of keen and safe healthcare systems using ML and unconventional security method predicted in the mixing of optimum storing and data security layer uphold security and privacy. Diverse procedures like concealing encryption, action supervising, granulated access control, active data encryption and conclusion point authentication have been unified. The fusion of four-layer healthcare archetype looks extra effective illness investigative big data system.

\section{REVIEW OF LITERATURE}

Coronary artery disease is a general enduring sickness, similarly predictable as an ischemic heart illness that is a cardiac dysfunction caused by the scarcity of blood amount to the heart and take a life of limitless individuals every single year. In current eons, coronary artery disease levels earliest and first amid the biosphere's topmost ten reasons of demise.

This investigation instructs a programmed cataloging method for phonocardiograms by means of deep and ensemble learning methodologies through a SavitzkyGolay filter [6]. This research paper contributes a multicentroid diastolic duration model for the Hidden SemiMarkov Model (HSMM) built for heart sound segmentation.

The centroids are calculated by hierarchical agglomerative clustering of the neighboring diastolic period rates using Ward's technique till focus of clusters is initiated at least a systolic interval apart. The numerous peak distributions produce a high-pitched slope of probability from place to place the expected centroids and advances the discriminability of analogous interpretations [7]. Deep belief networks are a type of the deep learning methods, choosing the significant characteristics to lessen the high dimensionality via stack of Restricted Boltzmann Machine and optimize to boost weights and diminish the renovated feature error. Thereby, removing the qualities that entertain extra reconstruct error and only use only less constrict error showing the experimental enhancements of results in Virtual Screening outcomes [8].

This scientific study's objectives are classifying the data mining methods and systems that are generally realized in exploring linked to numerous disease risk prediction archetypes also detecting the accuracy of these systems entails many techniques, but this scientific work is aiming at global precision that is evaluated by the over-all sums of precisely predicted outcome on top of the entire amount of estimates. A methodical review and creative writing about five databases initiate 170 articles, of which 7 items were nominated in the ending activity. This evaluation instituted that utmost prediction patterns used classification method, with an emphasis on decision tree, neural network, support vector machines, and Naïve Bayes algorithms everywhere heart-associated illness is usually researched [9]. This survey paper primarily reports on the optimization procedures of Particle Swarm Optimization (PSO), Gravitational Search Algorithm (GSA), and Ant Colony Optimization (ACO), that 
remain exploited recently with Fuzzy Logic (FL) to recuperate the accomplishment of the optimization processes [10].

The 2014 DARPA notice of the plug-in detailed, "Plasticity turns to neutralize formerly had notions that the matured brain is a "completed" object that can be statically charted. Due to elasticity, scientists are cheerful that the brain can be qualified or cured to reestablish usual functionality ensuing hurt or the inception of neuropsychological sickness."

Cognitive remedy is of progression creditable, then who is successful in describing "regular workability" and for what determination? [11].

Useful paper as contents are with Introduction, Discussions, BERLIN Agreement, Plenaries, Plenary Sessions, Pre-Workshops Research, Education, Traditional Healing Systems, Orals Clinical Care, Medicine and Symposia, Posters, Various Topics, Author Index Workshops, etc., [12].

\section{FUTURE TRENDS AND ENHANCEMENTS IN \\ healthcare Data Predictive analytics using Soft COMPUTING TECHNIQUES IN DATA SCIENCE}

Data Science and Data mining turn into more and more generic together in the private and public zones. Businesses such as banking, insurance, medicine, and retailing normally use data mining to reduce charges, boost exploration, and rise auctions. So, data science and mining will be progressively valuable in the upcoming period ahead.

Data science is yet similar to software growth and its extra multifaceted venture nurture it further into its viability to decline.

An undertaking might be required externally for our comfort regions to obtain the chances and disputes that this digital gold fetches. As the volume of data continues to grow, SC algorithms get elegant and our computational skills advances, and the requirements to adapt. Confidently, by generating a robust situation for using data science in our establishment and be virtuously equipped for what the approaching spell ahead fetches.

The three entangled inclinations of growing volumes of data, better-quality SC procedures and improved computing possessions are influencing the data science field in electrifying ways.

Improvement of valuable visions on artefacts/corporate from data and its mined information. Practically all corporations today handle data-driven choices by using one method or additional methods. And if they don't, they will have to resolve it in the nearest imminent time ahead.

Data Science is on a temporary approach and it is in relationships of how the modern data technologies are cast off in resolving commercial complications for a premeditated benefit. At the close time to come, data scientists will handle their occupation more contrarily. As Big Data, Algorithmic economics, IoT, and Cloud endure in developing as a mainstream amid of universal establishments, trades drive lingering becoming mostly accustomed to the new inexpensive policies and stay ahead of the curve. The two furthermost outstanding facts of this evolution are amplified mechanization of data methods and distribution of instant logical results.
The forthcoming of SC in healthcare is enormously auspicious with a numberless of forward-thinking SC practices such as deep reinforcement learning, one-shot learning, and capsule network be in collaboration with clinicians to allow innovative data in biomedicine and healthcare that SC can be omnipresent and unseen in the future health care areas realizing novel data from entire sources. Furthermore, there requires a crossing point between clinicians with data and computer scientists with analytics to assure a data-to-information continuum and a knowledge-tointelligence transfer.

The Future of SC: Augmented and Virtual Reality Blockchain and Cybersecurity Cloud Computing Deep Learning and New Types of Learning (Capsule Network) (Third Wave) (Deep Cognition) Internet of Everything Other Key Concepts: Virtual Assistants and Bots, Swarm Intelligence, Quantum Computing - Neuromorphic Computing - Hypergraph Databases - Brain-Computer Interface

Finally, need to disseminate a human-machine synergy via a clinician-data scientist collaboration without hubris to push future healthcare and medicine to the highest echelon.

\section{Measures ANd Metrics For HealthCare Data Predictive ANAlytics}

Healthcare analytics is specifically based on data, and data sets that comprises of massive volume of medical data, numerous measurements, economic data, arithmetical data, demographics of definite residents, and insurance data, a limited data declared, collected from many healthcare data bases. A list of the maximum healthcare data sets that is made up of both free healthcare data sets and business data sets applied for arithmetical investigations are given below. Healthdata.gov, World Health Organization, data.gov, The Human Mortality Database (HMD), Data and Tools of the National Center for Health Statistics, openFDA, The Big Cities Health Inventory Data Platform, Medicare.gov, US Census Bureau, National Cancer Institute, etc.

1. Volume Metrics, 2. Revenue Leakage Metrics, 3. Utilization Metrics, 4. Quality Metrics, 5. Financial Metrics, 6. Incidents Metrics, 7. CMS Program Performance, 8. Cohorts Metrics, 9. Clinical Metrics are the few metrics need to be predicted.

1. Volume Metrics: refers to the stream of patients to our facility. This includes:

a. Total number of patients by section, by specialism

b. Time of appointment and length of visit

c. Tracking origin of and number of inbound referrals

2. Revenue Leakage Metrics denotes the data associated to the flow of patients over our ability with missing revenue prospects. This consist of:

a. Negated arrangements owed to patients not disclosing in the lead

b. Tracking the amount of outbound appointments as it should be unconfirmed specialties

c. Invalid or reorganized engagements suitable for member of staff inaccessibility.

3. Utilization Metrics speak of the patient traffic and utilization of supplies caused by every sections separately, expert knowledge, and good turn dealer. This takes in: 
a. Appointments accomplished per doctor

b. Surgeries carried out per surgeon

c. Appointments booked per section and special study.

4. Quality Metrics imparts to the effectiveness of our quality, safety, and access initiatives. It is the most extensive data-set that will be tracked on our dashboard. This comprises:

a. Member Satisfaction

b. Patient Satisfaction and Engagement

c. Post-Treatment metrics

i. Readmission rates for specific diseases (e.g. heart failure, pneumonia, etc.)

ii. Clinical outcome rate statistics

iii. Occurrence of hospital acquired infections

iv. Clinical error ratios

v. Outpatient wait time

vi. Mortality

vii. Safety of care

viii. Timeliness of care

ix. Patient experience

x. Effectiveness of care

xi. Efficient use of medical imaging

5. Financial Metrics is further magnificently modified and aimed on the fiscal performance of our healthcare facility. This embraces:

a. Revenue made per doctor

b. Revenue created by section

c. Revenue caused by specialty

d. Revenue/expenses per doctor/section/ specialty.

6. Incidents Metrics comprise unpremeditated effects or lateral outcomes of hospital events, including unsolicited conditions like sepsis, postoperative respiratory failure, pulmonary embolisms, hemorrhages, and further consequences or contaminations. This metric deals with the capability of healthcare specialists to deliver wide-ranging, superior care to patients deprived of eliciting an adversative response.

7. CMS Program Performance Value-based programs targeting to cut down the total healthcare expenses and advance care eminence throughout deals with monetary prizes for enhancement on a variation of clinical and quality metrics irrespective of the facility aimed for or establishment. 8. Cohorts Metrics Inspect the activities and functioning of clusters of manipulators associated by joint qualities. A cohort is a cluster of manipulators who apportion a generic trait that is branded in the report by an Analytics facet. The Cohort Analysis report permits to segregate and evaluate cohort conduct.

9. Clinical Metrics are operational metrics, or key performance indicators or data points that make available awareness into operative occurrences with the uses as: refining methods within and establishment of associations with promoters.

Statistical Metrics are quantitative measurements. This includes:
a. R-squared
b. Average error
c. Mean Square Error (MSE)
d. Average absolute error
e. Median absolute error
f. Tracking Signal
i. Mean Absolute Deviation
ii. Mean Absolute Percentage Error
g. Control Chart.

Evaluation Metrics that desires to be included are available in the following:
a. Accuracy
b. Sensitivity \& Specificity
c. True Positive, True Negative, False Positive, and False Negative
d. Consistency
c. Precision \& recall
e. Chances of illness
f. Quality Ratings
h. Positive Predictive Value, Negative Predictive Value
i. ROC Curve - Receiver Operating
Characteristic Curve \& AUC - Area Under the Curve
j. $\quad$ Lift and Gain charts
k. Percent correction classification (PCC)

\section{Confusion matrix}

A Dashboard is a concise, clearly legible, frequently graphical exhibit of the key performance indicators (KPI) an administration team requires to proctor repeatedly. It delivers a single view of statistics commencing from corner to corner of an organization and portrays it in an easily handy means.

Benefits of the Dash Boards in Healthcare includes the following - discerning wherever setbacks are and latent extents for revolution in consenting them to render nobler resolutions, advance the quality of concern, making available additional proficient resource management, decrease expenses and to augment the amount of work of the medicinal staff. Statistical investigation is vital in signification-based medication.

Dash Boards for the following are to be incorporated with:

a. Prediction Interval

b. Confidence Interval

c. Predictive Statistics

d. Predictive Inference

e. Predictive Analytics

f. Demands for Healthcare Services -

i. Percent Adjustment,

ii. 12 Months Moving Average,

iii. Trendline,

iv. Seasonized Forecast.

g. Healthcare Needs

h. Prediction Score.

\section{Conclusions and Future Enhancements}

At the end of this review, exploration core points were taken in performance to confer what is the one and only tangible subsequent step to be acquired in the direction of state-of-the-art diffusion of investigations ahead motivating the investigators to learn novel platforms and procedures: identify the status that seem dedicated to publicizing the discoveries of their efforts. Even though investigators are intensive on hypothetical contributions, a variety of broadcasting events are ensuing useful even if in n profitable manner. Still, what establishes real propagation (in terms of influence and return on savings) stay uncertain. Investigators 
want superior and vibrant supervision on how worthiest to propose, source, and enable their broadcasting events.

Experienced, knowledgeable and skilled human assets are a prime qualification for any established development. All traditional healthcare systems are handling a stern disaster of manpower growth, deployment and headship. There is no appropriate guidance automated for outdated doctors. Hypothetical and other establishments are befalling track with deprived financial funding's, scheduling, organization, measuring, forecasting and governance.

It is trusted that the evidences provided will support the researcher in the time ahead to escalate the complications of reviewing remedial healthcare and the problems integral in this form of education. The future developments are formulated in terms of current ages is to be inflicted with defined and essentially aids examiners to design scientific prosecutions of remedial healthcare that would esteem mutually the perceptions of the time-honored conventional, resent and modern systems of medicine, healthcare correspondingly, those of modern clinical tribunal techniques.

\section{REFERENCES}

[1] Qiang Yang and Xindong Wu, "10 Challenging Problems in Data Mining Research", International Journal of Information Technology \& Decision Making, vol. 05, No. 04, pp. 597-604, 2006.

[2] David Crockett, "Using Predictive Analytics in Healthcare: Technology Hype vs Reality", An Executive Report in Health Catalyst, 2017.

[3] Veena H Bhat, Prasanth Rao, Shiva Krishna, P. Deepa Shenoy, Venugopal K R, and Lalit M Patnaik, "An Efficient Framework for Prediction in Healthcare Data Using Soft Computing Techniques", Proceedings of Advances in Computing and Communications - First International Conference, ACC 2011, Part III, July 22-24, 2011, Kochi, India, \& Communications in Computer and Information Science, vol. 192, pp.522-532, July 2011.

[4] Antonis S. Billis and Panagiotis D. Bamidis, "Employing Time-Series Forecasting to Historical Medical Data: An Application Towards Early Prognosis within Elderly Health Monitoring Environments", Proceedings of the 3rd International Conference on Artificial Intelligence and Assistive Medicine AIAM'14, August 18 $8^{\text {th }}, 2014$, Aachen, Germany, vol.1213, pp 31-35, 2014.

[5] Yap, Bee Wah, Mohamed, Azlinah H, Berry, Michael W. (Eds.), Proceedings $4^{\text {th }}$ IEEE International Conference on Soft Computing in Data Science SCDS 2018, August $15^{\text {th }}-16^{\text {th }} 2018$,
Bangkok, Thailand. Communications in Computer and Information Science, 2019 Springer Nature Switzerland AG, 2019.

[6] Jimmy Ming-Tai Wu, Meng-Hsiun Tsai, Yong Zhi Huang, SK Hafizul Islam, Mohammad Mehedi Hassan, Abdulhameed Alelaiwi, Giancarlo Fortino, "Applying an Ensemble Convolutional Neural Network with Savitzk-Golay Filter to Construct a Phonocardiogram Prediction Model”, Applied Soft Computing, ELSEVIER, ScienceDirect, vol 78, pp 29-40, May 2019.

[7] Alex Paul Kamson, L. N. Sharma, S. Dandapat, "MultiCentroid Diastolic Duration Distribution based HSMM for Heart Sound Segmentation", Biomedical Signal Processing and Control, ELSEVIER, ScienceDirect, vol 48, pp. 265-272, February 2019.

[8] Maged Nasser, Naomie Salim, Hentabli Hamza, and Faisal Saeed, "Deep Belief Network for Molecular Feature Selection in Ligand-Based Virtual Screening", Data Science, AI and IoT Trends for the Fourth Industrial Revolution, International Conference of Reliable Information and Communication Technology, Kuala Lumpur, Malaysia, on July 23-24, 2018. Advances in Intelligent Systems and Computing, Springer Nature Switzerland AG 2019, vol. 843, pp 3-14, 2019.

[9] Wan Muhamad Taufik Wan Ahmad, Nur Laila Ab Ghani and Sulfeeza Mohd Drus, "Data Mining Techniques for Disease Risk Prediction Model: A Systematic Literature Review", Data Science, AI and IoT Trends for the Fourth Industrial Revolution, International Conference of Reliable Information and Communication Technology, Kuala Lumpur, Malaysia, on July 23-24, 2018. Advances in Intelligent Systems and Computing, Springer Nature Switzerland AG 2019, vol. 843, pp 40-46, 2019.

[10] Fevrier Valdez, Patricia Melin and Oscar Castillo, “A Survey on Nature-Inspired Optimization Algorithms with Fuzzy Logic for Dynamic Parameter Adaptation", Expert Systems with Applications, Elsevier, ScienceDirect, vol.41, no. 14, pp 6459-6466, 15 October 2014.

[11] Dr Marc Siegel, "Brain Implants: Will they be used to Heal or for Control?”, Opinion, The Hill, 22/10/2018, 2019 Capitol Hill Publishing Corp., A Subsidiary of News Communications, Inc, 2019.

[12] Meeting Abstracts, BMC Complementary and Alternative Medicine, World Congress Integrative Medicine \& Health, Berlin, Germany, 3-5 May 2017, vol 17(Suppl 1):322, pp 1165, 2017. 